\title{
The Moderating Effect of Emotional Intelligence on Leader's Job Performance
}

\author{
Yunfang Ma ${ }^{1, a}$, Dan Chang ${ }^{1, b}$, Lan Teng ${ }^{1, c}$, Qiwen Du ${ }^{1, d}$ \\ ${ }^{1}$ Beijing Jiaotong University, Beijing, China \\ ayunfangm@bjtu.edu.cn, bdchang@bjtu.edu.cn, \\ caugtl@hotmail.com, d14113135@bjtu.edu.cn
}

Keywords: emotional intelligence, leader, job performance, moderating effect.

\begin{abstract}
Based on the existing emotional competency model, this paper starting from four dimensions including leader's self-awareness, self-management, social awareness and relationship management. We combined with the leader's personal performance and team performance put forward eight hypotheses. And we found out that emotional intelligence of leaders significantly influence individual performance and team performance, which also proved the leader's emotional intelligence affects the whole team's work efficiency. So that on the basis of their own characteristics, different leaders should control emotional problems encountered in the management process from self-awareness, self-management, social awareness and relationship management, to further improve the work performance.
\end{abstract}

\section{Introduction}

In real life, we often find that the performance of leaders with the same educational background and the same skills has great difference, which shows that non intelligence factors affect the leader's job performance. With the increasing pressure of social competition today, leaders with higher levels of emotional intelligence are more likely to achieve high performance through assessing emotions, adjusting their emotions and using their emotions. The development of an enterprise depends on the leader's decision, the leader's decision is often affected by many factors, which influence the emotional intelligence occupy a large proportion, but because the role of emotion leader decision can make the development of enterprises has a different direction. In the existing research, the leader's emotional competence model with four dimensions has been established, as shown in Figure 1, which contains 4 dimensions: leader's self-awareness, self-management, social consciousness and relationship management. The leader's job performance is usually divided into individual performance and team performance, which is divided into the two dimensions of research, this paper will influence on the working performance of the emotional intelligence of leaders on the basis of this, in order to have a better assessment of the leader of their own, adjust their own emotions to improve work performance.

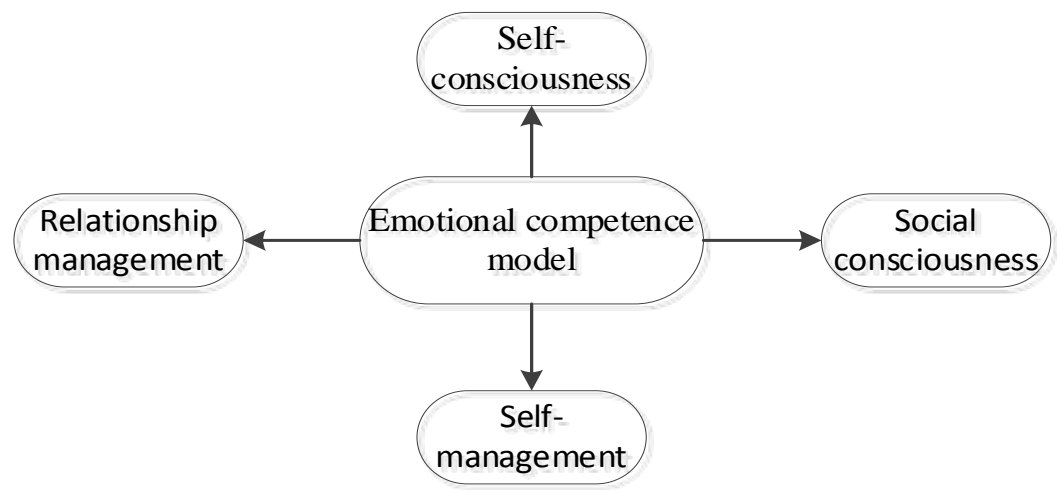

Figure 1 leader's emotional competence model with four dimensions 


\section{Literature review and hypothesis proposed}

Different types of leadership have different effects on different dimensions of organizational citizenship behavior. Liang Qiaozhuan and Ma Xianwen [1] used multiple linear regression method, proposed between the boss of leadership style on distributive justice and organizational citizenship behavior of different dimensions of regulation of the four hypotheses based on the control of job satisfaction, through factor analysis and regression analysis to create dimensions of distributive justice and organization the various dimensions of citizenship behavior has no significant moderating effect, and the bosses concerned dimensions of distributive justice and loyalty relationship between maintenance no moderating effect on the relationship between distributive justice and OCB in overall and three other dimensions have significant moderating effect, through this research, can be obtained in relation to the distribution between justice and when OCB regulation, leaders should pay more attention to the interpersonal relationship between employees and the feelings of each other, but this The study only takes job satisfaction as an intermediate variable, and whether there are other intermediary variables on the basis of social exchange theory remains to be further studied.

Leadership styles also have positive or negative moderating effects on the crossover of R \& D personnel. Zhang Hualei, Yuan Qinghong et al. [2] put forward the core self-evaluation, leadership style and R \& D personnel cross influence each other and the five assumptions that R \& D personnel adjustment, core self-evaluation and authorization of leadership have a positive effect on R \& D personnel cross-border behavior; authorized leadership and instructions leadership are positive and negative regulation of $\mathrm{R} \& \mathrm{D}$ personnel core self-evaluation on its cross-border behavior effect. This research provides practical guidance for border management of R \& $\mathrm{D}$ teams. Similarly, this article does not refine leadership style into emotional intelligence.

Based on the emotional competency model, it can be studied in depth. Hu Xinbu and Yuan Zhiping [3] used factor analysis method, based on literature analysis and interviews, research and design leaders emotional initial Competency Scale, combined with factor analysis and reliability analysis identified 4 dimensions of emotional leadership competency Netlon syndrome, respectively, self-management, self-consciousness social awareness and relationship management, and to determine the characteristic elements of each dimension, different dimensions of the factors extracted by the number were 2, 3, 2 and 4 compared to the previous study, a decrease of 7 , we build a Chinese scenario leader emotional competency model. But the results obtained in this paper can not only know as the main members of the group leaders should have what qualities and skills, can also carry out training plan on the basis of research results, to make up for, enhance the leader's ability and quality. However, after the construction of the model, there is lack of a further evaluation and application of the various indicators of the model, so that the actual implementation of the model there are some problems.

The performance of leaders can be divided into individual performance and team performance. In the previous study, the different leadership style into care leading dominant leadership and initiative dimension of leadership, and directive leadership and the authorized leadership, different types of leadership to influence organizational performance will have different effects. Although these articles on the effects of different leadership styles on the performance or there is a lot of discussion between the different dimensions of organizational culture and regulation, but all these discussions have ignored the impact of emotional intelligence on different leader leaders, regulation based on the existing leader emotional competency model on emotional intelligence of different leaders the individual performance.

There are 4 dimensions in the emotional competence model: self-awareness, self-management, social awareness and relationship management. In view of the relationship between the four dimensions and the leader's personal performance and the relationship between these eight dimensions, the following hypotheses are put forward:

Hypothesis 1: leader's self-consciousness is positively related to leader's personal performance;

Hypothesis 2: leader's self-consciousness is positively related to leader's team performance; 
Hypothesis 3: leader's self-management is positively related to leader's personal performance;

Hypothesis 4: leader's self-management is positively related to leader's team performance;

Hypothesis 5: the leader's social consciousness is positively related to the leader's personal performance;

Hypothesis 6: leader's social consciousness is positively related to leader's team performance;

Hypothesis 7: leader's relationship management is positively related to leader's personal performance;

Hypothesis 8: leader's relationship management is positively related to leader's team performance.

\section{Research Method}

According to the specific characteristics of the four dimensions of emotional competence model to design the questionnaire, the two dimensions of self-consciousness for self-evaluation and self-confidence, evaluation of the advantages and disadvantages of personal understanding can objectively and accurately, and is confident of individual ability and correct judgment and grasp the three dimensions of self-management characteristics; respectively, self-control, and actively adapt to the optimistic and effective self-control can avoid the bad mood caused by impulse, and can correctly treat the pros and cons, the so-called active adaptation is spontaneous to change their own situation according to the requirements of the environment, and optimistic honesty is the reasonable way of looking at things; two social characteristics the dimensions of consciousness and sense of organization for empathy, empathy can stand in each other's point of view to consider the issue, can be effective coordination between the two groups. Fabric consciousness is for the reasonable sentiment organization environment of life; four characteristics of relationship management dimension respectively, good communication and teamwork, training others and the courage to change, need to have good communication to attract and persuade others will, team needs to maximize the rational use of available resources and strength of team or organization to pursue collective the target of interest, raise the overall quality of others by changing the groups, the efficiency of the organization, and the courage to change is one of the important means to move forward.

In order to determine the leader's personal performance and team performance, two sets of questionnaires has been designed. One of them is used to measure the leader's personal performance, which is filled in by the leader himself. The measurement of individual performance scale using Bas A.S. Koene. Another one is used to measure the team performance of leaders, using Chiu, Warren C.K. scale. The questionnaire is full by secret ballot of the employees, so as to correctly measure the leader of the team performance.

We selected ten enterprises in Beijing city to send out questionnaires. There are 10 questionnaires of individual performance and 300 questionnaires of team performance. Every enterprise received 30 copies. Each enterprise conducts a survey on one of the specific leaders. In order to ensure the reliability of the data, we adopted secret ballots to fill questionnaires on the spot, so as to ensure the amount of questionnaires, and finally collected and collated the data.

The independent variables identified in this study are leaders' self-consciousness, self-management, social consciousness and relationship management. The dependent variables are the leader's individual performance and team performance.

The data were analyzed by multiple linear regression analysis used by spss20 and test F-value and $R^{2}$, and obtained significance. Finally, verified the hypothesis

\section{Result analysis}

Through the analysis of the experimental results, the experimental results are more significant, and concluded that the eight assumptions are established, that is to say, the leader's emotional intelligence has a significant impact on the leader's job performance. Analysis of the reasons, first, 
the leader is able to objectively and accurately understand their own strengths and weaknesses, and correct judgment of individual ability, not only has a significant effect on individual performance and team performance of leaders to improve; secondly, to properly manage emotions, the mood is kept in appropriate and timely state based on emotional self-perception. Can master their own emotions, people can quickly get out of the low tide of life, enhance the self-restore vitality, so as to improve the work performance; third, in the work process, if thought leaders can reasonable stand in the perspective of others and deal with the problem, will greatly improve the job satisfaction of employees has a positive effect on job performance; finally, interpersonal relationship is to manage the emotions of others art, in the complex group, can get along well with others "master this Person with ability is usually the leader of society, and naturally, job performance will be improved.

\section{Conclusion}

Through this study it can be significantly affected the individual performance and team performance emotional intelligence of leaders, which also proved the leader's emotional intelligence affects the whole team's work efficiency, so that different leaders should control their own characteristics, from self-awareness, self-management, social awareness and relationship management, reasonable and effective control the emotional problems encountered in the management process, to further improve the work performance.

\section{Acknowledgement}

This work was partially supported by Beijing Logistics Informatics Research Base.

\section{References}

[1] Qiaozhuan Liao, Xianwen Ma, Yingbo Huang, Hui Sun, Zhizhong Yin. A study on Moderating Effects of Supervisor's Leadership Style on the Relationship between Perceived Distribution Justice and Organizational Citizenship Behaviors [J]. Journal of Industrial Engineering/ Engineering Management. 2007, 3 (21) 9-13.x

[2] Hualei Zhang, Qinghong Yuan, Zhen Wang, Yong Huang, Influence of Core Self-Evaluations and Leadership on R \& D Professionals ' Boundary—Spanning Behavior [J]. Chinese Journal of Management, 2014, 11(8) 1168-1176.

[3] Xinbu Hu, Zhiping Yuan, Hong Chen, Linna Hou, The Empirical Study of Leader' S Emotional on the Development Competence Model for Higher Cadres of Shaanxi Province in China [J]. Soft science. 2014.6(28) 85-89.

[4] Hui Li, Gang Ding, Xinjian Li. The Impact of Leadership on Employee Innovation Behavior in the Context of China - The Perspective of Paternalistic Leadership Ternary Theory [J]. Chinese Journal of Management.2014,7(11),1005-1013. 